\title{
(Epidemiological Analysis of Varicella in Dalian from 2009 to 2019 and Application of Three Kinds of Model in Prediction Prevalence of Varicella冈Study Design: Cross Sectional Study
}

\section{Tingting Cheng}

Dalian Medical University

\section{Yu Bai}

Dalian Center for Disease Control and Prevention

\section{Xianzhi Sun}

Dalian Medical University

Yuchen Ji

Dalian Medical University

\section{Fan Zhang}

Dalian Medical University

Xiaofeng Li ( $\square$ Ixf_chen@dmu.edu.cn )

Dalian Medical University

\section{Research Article}

Keywords: varicella, epidemic characteristics, $\mathrm{GM}(1,1)$ model, Markov model GM(1,1)-Markov model

Posted Date: November 16th, 2021

DOI: https://doi.org/10.21203/rs.3.rs-1026752/v1

License: (c) (1) This work is licensed under a Creative Commons Attribution 4.0 International License.

Read Full License 


\section{Abstract}

Objective区This study described the epidemic characteristics of varicella in Dalian from 2009 to 2019, explored the fitting effect of Grey model first-order one variable( $\operatorname{GM}(1,1))$, Markov model, and $\operatorname{GM}(1,1)$ Markov model on varicella data, and found the best fitting method for this type of data, to better predict the incidence trend.

Methods: In this study, the epidemiological characteristics of varicella from 2009 to 2019 were analyzed by epidemiological descriptive methods. Using the varicella incidence data from 2009 to 2018, predicted 2019 and compared with actual value. First made GM $(1,1)$ prediction and Markov prediction. Then according to the relative error of the $\mathrm{GM}(1,1)$, made $\mathrm{GM}(1,1)$-Markov prediction.

Results: This study collected 37223 cases from 2009 to 2019 . The average annual incidence was $50.56 / 100000$. Varicella occurred all year round, it had a bimodal distribution. The number of cases had two peaks from April to June and November to January of the following year. The ratio of males to females was $1.167: 1$. The 4 to 25 accounted for $60.36 \%$ of the total population. The age of varicella appeared to shift backward. Students, kindergarten children, scattered children accounted for about $64 \%$ of all cases. The $\mathrm{GM}(1,1)$ model prediction result of 2019 would be 53.6425 , the relative error would be $14.42 \%$, the Markov prediction result would be 56.2075 , the relative error would be $10.33 \%$, and the Gray(1,1)-Markov prediction result would be 59.508 . The relative error would be $5.06 \%$.

Conclusions: Varicella data had its unique development characteristics. The accuracy of GM $(1,1)$ Markov model is higher than GM(1.1) model and Markov model. The model can be used for prediction and decision guidance.

\section{Background}

Varicella is an acute infectious disease caused by the first infection with varicella-zoster virus (VZV). The incubation period is about 2 weeks, and some cases can reach 3 weeks. It is highly contagious from the day before the rash to full scab healing. The main transmission route is respiratory droplets or direct contact infection. Varicella patients are the only source of infection.

Varicella has a high infection rate in China. Varicella usually heals on its own. Individuals, infants, and adults with low immune function may be serious ${ }^{1}$. VZV is a teratogen and causes congenital varicella syndrome (CVS) ${ }^{2}$ Moreover, VZV is a common cause of stroke ${ }^{3}$. Varicella has complicated complications and causing serious economic burden. Vaccines are the most economical method ${ }^{4}$.

The incidence of varicella in China ranks first among vaccine-preventable infectious diseases ${ }^{5}$. Studies found that children were vaccinated with varicella vaccine, the protective effect of the vaccine declined year by year, varicella would break out again ${ }^{6}$. Some severe breakthrough varicella can occur, but it does not seem to be common ${ }^{7}$. Research shows, the incidence of varicella has shown an overall upward trend ${ }^{8,9}$. Adults infected varicella easier and more dangerous. In many places, the age of varicella had 
been found to shift backward ${ }^{10}$. Studies have found that adults in tropical and subtropical countries were more likely to suffer from varicella than in countries with mild climates ${ }^{11}$. Many factors like air pressure, temperature, humidity, rainfall, and other subtle factors can have a complex impact on the incidence of varicella ${ }^{12}$. There are differences in different regions, and the incidence of varicella is different. Find a model suitable for the varicella data in Dalian, explore the fitting effects of different models on the data, and provide a theoretical basis for varicella prediction and health decision-making.

The gray model was proposed by Professor Julong Deng in $1982^{13}$. It is a widely used data prediction model. It can suit various data types. It seeks valuable information by fully extracting and developing small samples and poor information data. And it generates predictions to demonstrate the direction of the system. Grey model first-order one variable $(\operatorname{GM}(1,1))$ is the core of the gray model and it was used widely. While in varicella prediction, the GM $(1,1)$ was less used.

The Markov chain theory was proposed by a famous mathematics scientist A.A. Markov in 1906, The Markov model was maturely used in the economic field and played an important role in medical dynamics prediction ${ }^{14}$, Markov prediction means that in the process of data transfer, under the action of a certain factor, their state probability depends on the previous result, and the probability law of the Nth result depends only on the result of the ( $\mathrm{N}-1)$ th experiment. And has nothing to do with the earlier results, the process is a random process ${ }^{15}$, Predict the state of the process at the next moment and the next few moments through the law of change between the states of the random process at different moments ${ }^{16}$.

The general experience of the Grey forecast dynamic model believes that its prediction accuracy for random, long-term, and volatile historical data is low, and it is mainly suitable for reflecting the overall development trend of the forecast ${ }^{17}$. While the Markov forecast is mainly based on the current state and the law of state transition predicts the possible state of the system in the future, generally through the transition probability matrix for prediction and decision-making. It can just make up for the limitation of the gray model. Therefore, try to use the Markov principle to fix the gray prediction value. Combine the advantages of two models to establish a Gray(1,1)-Markov prediction model ${ }^{18}$.

In this paper, two parts were included. First, an epidemiological description of varicella in Dalian from 2009 to 2019. Second, GM $(1,1)$ model, Markov model, and GM(1,1)-Markov model were established to fit the incidence of varicella and to explore the fitting effects of different models on varicella data.

\section{Materials And Methods \\ 2.1. Materials}

The data in this study was collected from Dalian Centers for Disease Control and Prevention (CDC). The varicella data from 2009 to 2019 includes the total infection number, age distribution, months distribution, and the occupation distribution. Among them, the occupation distribution in 2009 was 
missed. The GM $(1,1)$ model and Markov model was built with data from 2009 to 2018. The GM $(1,1)$ Markov model was built according to the result of the $\mathrm{GM}(1,1)$ model.

\subsection{Descriptive epidemiological analysis}

The epidemiological characteristics of varicella in Dalian from 2009 to 2019 was described by time and population. Statistical charts were adopted to intuitively describe the epidemic characteristic.

\subsection{GM $(1,1)$ model}

The data used from 2009 to 2018 as the original data, established a GM $(1,1)$ model and predicted the number of varicella in 2019 , and analyzed the accuracy of the $\operatorname{GM}(1,1)$ model prediction results.

\subsection{Markov model}

The data used from 2009 to 2018 as the original data. K-mean cluster was adopted to divide groups. The state transition matrix was used to predict the 2019 group matrix and Markov model prediction value.

\section{5. $\mathrm{GM}(1,1)$-Markov model}

The relative value between the predicted value of the $\mathrm{GM}(1,1)$ model from 2009 to 2018 and the true value was used as the original data. K-mean cluster was used to divide groups. The state transition probability matrix was calculated according to grouping. 2018, 2017, and 2016 were selected as the three most recent years from 2019. The three-step transition probability matrix was used to calculate the state interval of 2019. $\mathrm{GM}(1,1)$ model prediction value was combined to calculate $\mathrm{GM}(1,1)$-Markov model prediction value.

\subsection{Statistic software}

The epidemiological analysis of varicella data was collected and analyzed by Excel 2010 software. $R$ software 3.2.5 version was adopted to conducted $\operatorname{GM}(1,1)$ model prediction, the posterior difference ratio (C), and the probability of small error $(P)$ were used to evaluate the effect of prediction of the $\operatorname{GM}(1,1)$ model. Markov model and GM(1,1)-Markov model was implemented with the help of MATLAB 2015a software. Relative error was selected as the index to evaluated the accuracy of the models.

\section{Result}

\subsection{Epidemic characteristics}




\subsubsection{Time distribution}

The total number of reported cases of varicella in Dalian from 2009 to 2019 was 37223 . The incidence rate decreased and then increased on the whole. The lowest incidence rate was occurred in 2014(see Table1هFigure1). Varicella occurred throughout the year. The number of cases was increasing from February to May and August to December. From April to June, and November to January of the following year, there were more cases(see Figure2). Varicella cases showed obvious seasonality.

\section{Table 1}

The cases and incidence rate of varicella in Dalian from 2009 to 2019

\begin{tabular}{|cll|}
\hline Year & Cases & Incidence rate(1/100000) \\
\hline 2009 & 3502 & 57.96 \\
\hline 2010 & 3371 & 55.78 \\
\hline 2011 & 3709 & 55.44 \\
\hline 2012 & 2922 & 43.25 \\
\hline 2013 & 2684 & 39.93 \\
\hline 2014 & 2455 & 36.05 \\
\hline 2015 & 3139 & 46.09 \\
\hline 2016 & 2897 & 42.33 \\
\hline 2017 & 3895 & 55.75 \\
\hline 2018 & 4296 & 61.65 \\
\hline 2019 & 4353 & 62.68 \\
\hline
\end{tabular}

\subsubsection{Population distribution}

\subsubsection{Age distribution}

Varicella occurred any at age. The cases aged from 0 to 25 accounted for $92.46 \%$ of the total cases (see Table2). More cases aged from 5 to 10 (see Figure3).

Table 2

The varicella cases age distribution and proportion in Dalian from 2009 to 2019 


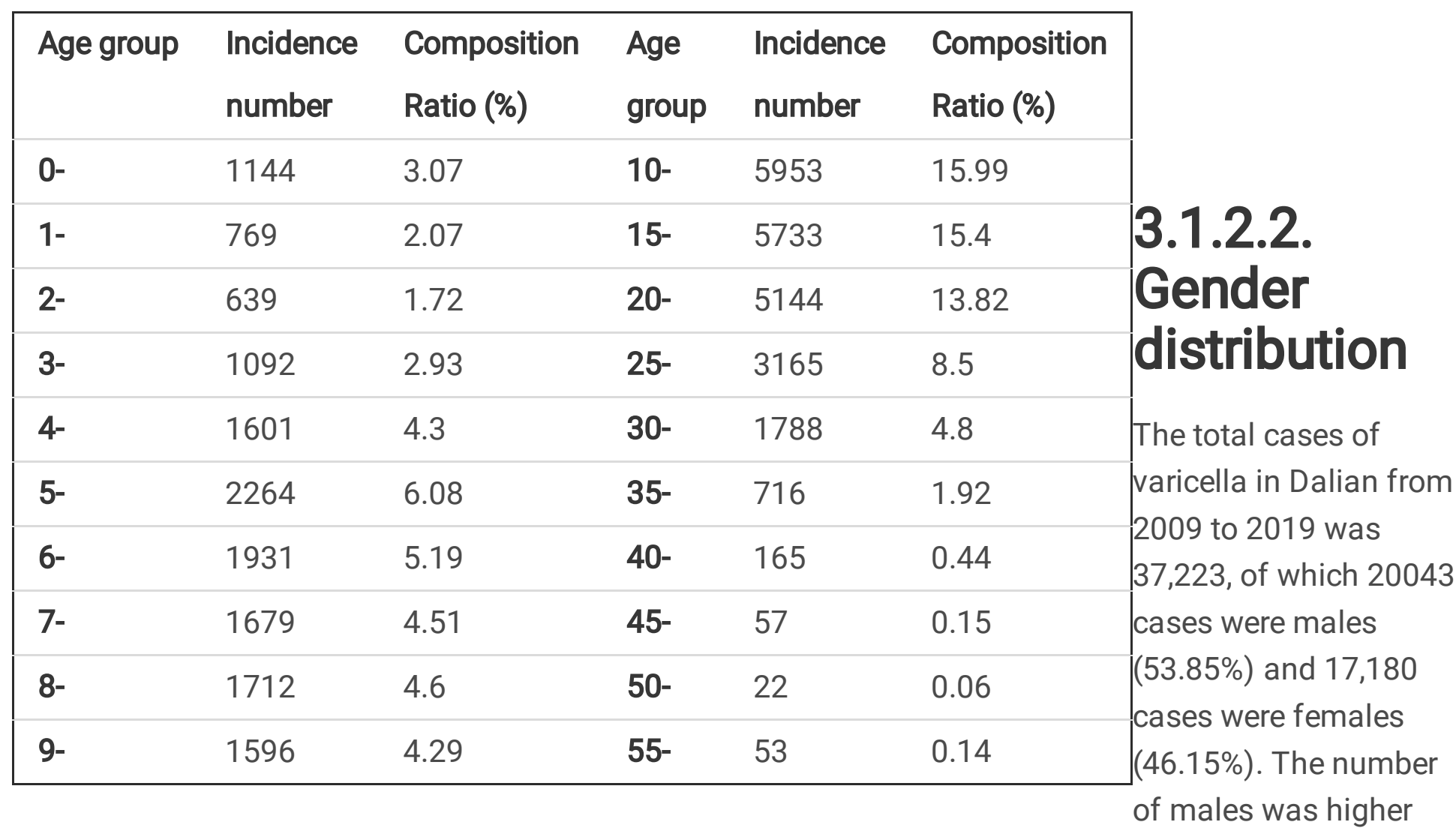

than that of females, and the ratio of total male to female was 1.17:1. (Figure4)

\section{2. $\mathrm{GM}(1,1)$ model}

Using R software for $\mathrm{GM}(1,1)$ model prediction. The incidence for 2019 was 51.3/100000(Table3), the posterior difference ratio $(C)$ was 0.987188 , the probability of small error $(P)$ was $0.3333 . a=-0.0114$ , $\mathrm{u}=45.3757$. Then the GM $(1,1)$ model was established as follows:

$$
x^{(1)}(k+1)=4038.28 e^{(0.52 k)}-3980.32
$$

Table 3

$\mathrm{GM}(1,1)$ model prediction results with $\mathrm{R}$ software

\subsection{Markov model}

\subsubsection{State division and state transition probability matrix::}




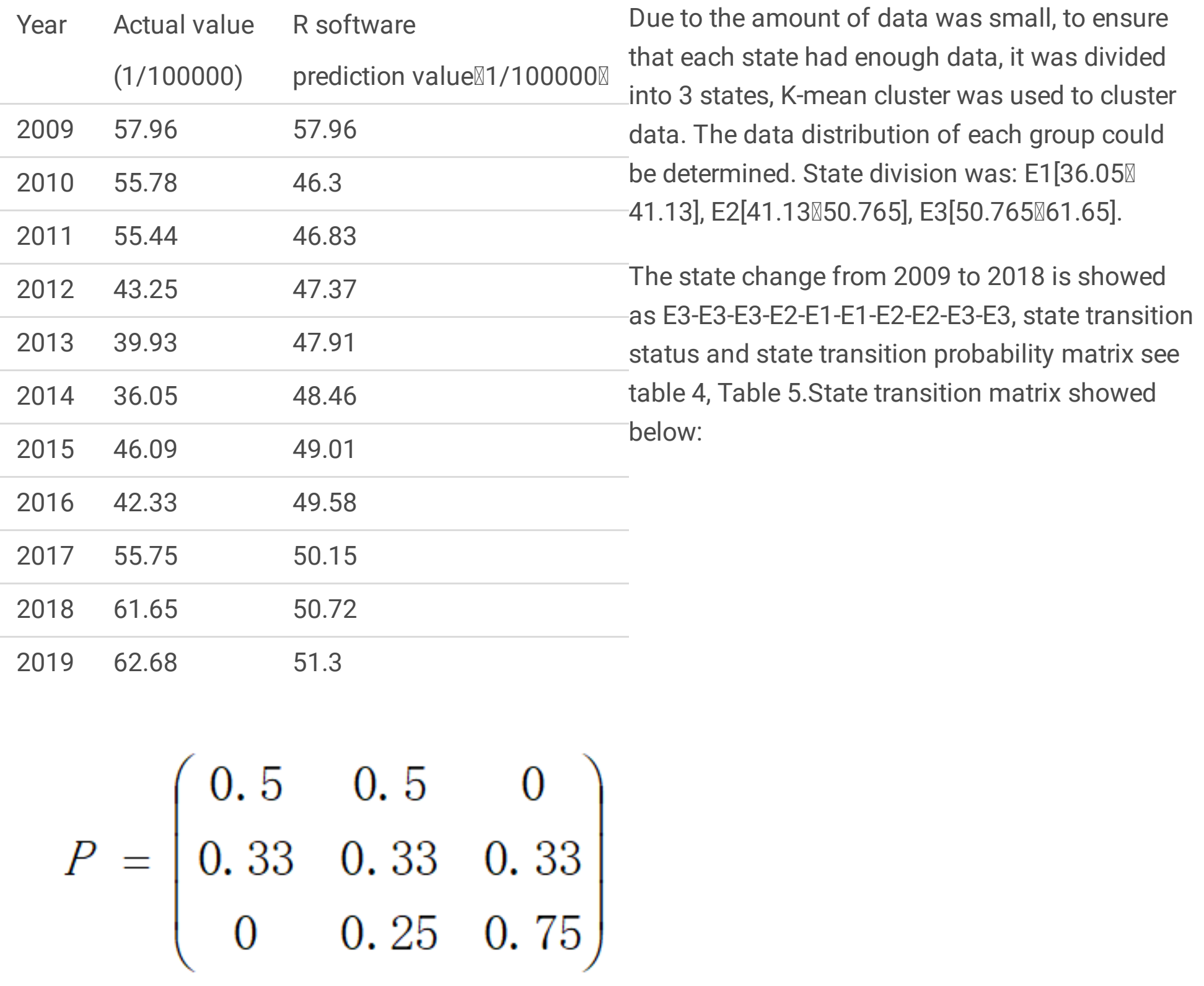

Table 4

State transition from 2009 to 2018

\begin{tabular}{|llll|}
\hline State & E1 & E2 & E3 \\
E1 & 1 & 1 & 0 \\
E2 & 1 & 1 & 0 \\
E3 & 0 & 1 & 3 \\
\hline
\end{tabular}

Table 5 


\begin{tabular}{|llll|}
\hline State & E1 & E2 & E3 \\
\hline E1 & 0.5 & 0.5 & 0 \\
E2 & 0.33 & 0.33 & 0.33 \\
E3 & 0 & 0.25 & 0.75 \\
\hline
\end{tabular}

\subsubsection{Use the state transition probability matrix to predict:}

With the help of MATLAB 2015a software, using the 2018 grouping matrix $(0,0,1)$ and the state transition probability matrix to predict. The result of the 2019 grouping matrix was $(0,0.25,0.75)$, the 2019 Markov model prediction was more likely in E3, so the value is: 56.2075

\section{4. $\mathrm{GM}(1,1)$-Markov model}

\subsubsection{State division and state transition probability matrix:}

Relative value was used to divide the state, relative value=actual value/ $\mathrm{R}$ software prediction value. $\mathrm{K}$ mean cluster was used to cluster data. The data distribution of each group could be determined. Divided all relative values into three states: underestimated, accurate, overestimated, namely E1 [1.1,1.22], E2[0.82,1.1], E3[0.74,0.82].The status changed from 2009 to 2018 is E2-E1-E1-E2-E2- E3-E2-E2-E1E1(Table6). State transition matrix see Table7

Table 6

State transition from 2009 to 2018

Table 7

State transition matrix 


\begin{tabular}{|lllll|}
\hline Year & Actual value & Predicted value & Relative value & State division \\
\hline 2009 & 57.96 & 57.96 & 1 & E2 \\
\hline 2010 & 55.78 & 46.3 & 1.2 & E1 \\
\hline 2011 & 55.44 & 46.83 & 1.18 & E1 \\
\hline 2012 & 43.25 & 47.37 & 0.91 & E2 \\
\hline 2013 & 39.93 & 47.91 & 0.83 & E2 \\
\hline 2014 & 36.05 & 48.46 & 0.74 & E3 \\
\hline 2015 & 46.09 & 49.01 & 0.94 & E2 \\
\hline 2016 & 42.33 & 49.58 & 0.85 & E2 \\
\hline 2017 & 55.75 & 50.15 & 1.11 & E1 \\
\hline 2018 & 61.65 & 50.72 & 1.22 & E1 \\
\hline 2019 & 62.68 & 51.3 & & \\
\hline
\end{tabular}

\begin{tabular}{|llll|}
\hline State & E1 & E2 & E3 \\
E1 & 0.67 & 0.33 & 0 \\
E2 & 0.4 & 0.4 & 0.2 \\
E3 & 0 & 1 & 0 \\
\hline
\end{tabular}

\subsubsection{Three-step transition probability matrix to prediction varicella in 2019:}

Select 2018, 2017, and 2016, the three most recent years from 2019. After one step (P1), two steps (P2), and three steps (P3), the state was transferred to 2019. Sum the column items, the maximum value was the state range, which the $2019 \mathrm{GM}(1,1)$ model predicted value located in, see Table8. 


$$
\begin{aligned}
& P(1)=\left(\begin{array}{ccc}
0.67 & 0.33 & 0 \\
0.4 & 0.4 & 0.2 \\
0 & 1 & 0
\end{array}\right) P(2)=P(1)^{2}=\left(\begin{array}{ccc}
0.5809 & 0.3531 & 0.066 \\
0.428 & 0.492 & 0.08 \\
0.4 & 0.4 & 0.2
\end{array}\right) \\
& P(3)=P(1)^{3}=\left(\begin{array}{ccc}
0.5304 & 0.3989 & 0.0706 \\
0.4836 & 0.418 & 0.0984 \\
0.4 & 0.4 & 0.2
\end{array}\right)
\end{aligned}
$$

Table 8

Status of the predicted values of the $\operatorname{GM}(1,1)$ model in 2019

\begin{tabular}{|lllllll|}
\hline Year & Initial state & \multicolumn{2}{l}{ transition steps } & E1 & E2 & E3 \\
\hline 2018 & E1 & 1 & (P1) & 0.67 & 0.33 & 0 \\
\hline 2017 & E1 & 2 & $(\mathrm{P} 1)$ & 0.5809 & 0.3531 & 0.066 \\
\hline 2016 & E2 & 3 & (P3) & 0.4836 & 0.418 & 0.0984 \\
\hline sum & & & & 1.7345 & 1.1011 & 0.1644 \\
\hline
\end{tabular}

The maximum sum value was in state $\mathrm{E} 1$, That was the $\mathrm{GM}(1,1)$ model prediction value will be in the $\mathrm{E} 1$ state, the prediction value of 51.3 was been underestimated, and the $\mathrm{GM}(1,1)$-Markov prediction value was $51.3^{*}(1.1+1.22) / 2=59.508$

\subsection{Models results comparison}

Three models results and comparison see Table9, Figure5 and Table10. GM(1,1)-Markov model fitted actual value better and had the lowest relative error of 2019.

\section{Table 9}

Models prediction results and actual value from 2009 to 2019 


\begin{tabular}{|lllll|}
\hline Year & Actual value & GM(1,1) value & Markov value & GM(1,1)-Markov value \\
\hline 2009 & 57.96 & 57.96 & 56.2075 & 55.6416 \\
\hline 2010 & 55.78 & 46.3 & 56.2075 & 53.708 \\
\hline 2011 & 55.44 & 46.83 & 56.2075 & 54.3228 \\
\hline 2012 & 43.25 & 47.37 & 45.9475 & 45.4752 \\
\hline 2013 & 39.93 & 47.91 & 38.59 & 45.9936 \\
\hline 2014 & 36.05 & 48.46 & 38.59 & 38.0411 \\
\hline 2015 & 46.09 & 49.01 & 45.9475 & 47.0496 \\
\hline 2016 & 42.33 & 49.58 & 45.9475 & 47.5968 \\
\hline 2017 & 55.75 & 50.15 & 56.2075 & 58.174 \\
\hline 2018 & 61.65 & 50.72 & 56.2075 & 58.8352 \\
\hline 2019 & 62.68 & 51.3 & 56.2075 & 59.508 \\
\hline
\end{tabular}

Table 10

Models results and relative errors of 2019

\begin{tabular}{|lllll|}
\hline Year & Actual value & $\mathrm{GM}(1,1)$ & Markov & $\mathrm{GM}(1,1)$-Markov \\
\hline 2019 & 62.68 & 53.6425 & 56.2075 & 59.508 \\
\hline Relative error $\% \bigotimes$ & & 14.42 & 10.31 & 5.06 \\
\hline
\end{tabular}

\section{Discussion}

There were 37223 varicella cases in Dalian from 2009 to 2019. The average annual incidence was $50.56 / 100000$. The high incidence was corresponding with our country's overall situation ${ }^{5}$. Varicella cases showed an upward trend just like other places $^{8,9}$. Varicella can occur all year round, mostly in spring and winter, seasonality is obvious. The two peaks of varicella cases were from April to June and November to January of the following year, showing a "bimodal distribution". This time the temperature change violently, people especially children who had low immunity prone to be infected. This time for children also studied at school. So, they can contact the outside world and other infected people easier.

In this paper, students, childcare, scattered children are the main disease groups. Most cases age from 5 to 10 . The cases aged from 0 to 25 accounted for $92.46 \%$ of the total cases. High-age cases account for a large proportion. In many places, the age of varicella had shifted backward ${ }^{10,19,20}$. Adults infected 
varicella became easy and common. Adult varicella is more harmful. Adults especially during pregnancy get more symptoms of varicella and more dangerous ${ }^{21,22,23}$. Many reasons like a vaccine, weather, climate, and others have a complication effect on the incidence of varicella $11,12,6$. We still need to find out the reason and solution to decrease the incidence.

The incidence ratio of varicella between men and women is $1.167: 1$, and the number of cases in men is higher than that in women. In many places, the varicella incidence rate, the male is higher than that of the female. And there is a statistical difference ${ }^{10,23,24}$. Student, childcare, scattered children accounted for $73.15 \%$ of the total of the cases. So varicella outbreaks were prone to occur in schools, kindergartens. These places require to Strengthen prevention and control.

The GM(1,1), Markov model, and Gray-Markov were used to predict the incidence of varicella in 2019. The predicted values were: 53.6425, 56.2075, and 59.508, and the relative errors of the true value in 2019 were: $14.42,10.33$, and 5.06 . From the results, the $\mathrm{GM}(1,1)$ model predicted the varicella cases were not ideal, and the model fitted poorly. Consider two follow reasons: 1The sample size was too small. 2 The sample size was too large. 3 The data was volatile. Concerning Tingting Zhang, Yanling Peng, and other documents $^{25}$, they collected 8 years of data from 2011 to 2018 and conducted $\mathrm{GM}(1,1)$ model prediction. The prediction level is excellent, and the amount of data is small, which is not the main reason for the unsatisfactory prediction. Besides, the $\mathrm{GM}(1,1)$ model is famous for predicting "small samples". There are also articles showing that the $\mathrm{GM}(1,1)$ model can be developed with only 4 models ${ }^{26}$, and the sample size was small, which was not the main problem. Research showed that fewer and recent modeling data have a good forecast effect ${ }^{27}$. When the number of modeling data between 4 and 10 . When the dimension was 4 , the prediction accuracy was the highest. With the increase of dimension, the precision did not increase ${ }^{28}$. But in this study, the number of $\operatorname{GM}(1,1)$ modeling data should be the same as Markov modeling data. More data, especially much old data had a bad influence on $\operatorname{GM}(1,1)$ model. The accuracy of the Grey model was inevitably decreased. The $\mathrm{GM}(1,1)$ model had a good predictive effect for sequences with short sequences and an upward trend ${ }^{29}$. The prediction results of the $\operatorname{GM}(1,1)$ model were generally smooth curves, reflecting long-term growth trends ${ }^{30}$, Poor fit effect for fluctuating data. Therefore, considering the large fluctuation of the sample was another reason for the unsatisfactory $\operatorname{GM}(1,1)$ prediction of this experiment. Consider further processing to improve forecast accuracy with this kind of data type. In this study, we combined $\mathrm{GM}(1,1)$ model and the Markov model to improve accuracy.

Markov predicted value in 2019 was56.2075, which was different from the actual value of 62.68 . The status division had a greater impact. Theoretically speaking, the longer the historical data, the more state division, the higher the prediction accuracy ${ }^{31}$. Generally, when there was less historical data, the number of states should be less, so that each state had more data. When there were more data, more sample falls into each state. The more the state increased, the more accurate the obtained prediction interval would be. In this study, we had less data, less state, and the transition change situation could not be better reflected. The state interval span was large, and the data prediction could not be effective and accurate. 
Besides, the state division based on the original data, the Markov model could not express the trend of continuous growth. Therefore, Markov prediction error would become larger and larger.

The Grey-Markov prediction result was better than the Grey model and Markov model prediction alone. The Gray model was widely used in predicting small sample data. It could describe the overall trend well, but it could not reflect data fluctuations well. The Markov model had a good predictive effect on fluctuating data. However, the data was less and the state was insufficient, which easily led to poor prediction results. And it could not describe monotonic change data well. Grey-Markov model forecasting can combine the advantages of the $\mathrm{GM}(1,1)$ model to predict small samples and the advantage of Markov's response to fluctuations, improving the prediction accuracy to a certain degree. GM(1,1)-Markov forecast achieved a relatively good forecast result with the small sample, volatility, and overall risen data. In further studies, GM(1,1)-Markov model can be used in similar medical data to predict disease development and formulate reasonable medical health measures. And we still need to continuously optimize the model and explore better ways to get better results.

\section{Conclusion}

The incidence of varicella fluctuated greatly and showed an upward trend as a whole,and the age of onset shifted backward. GM(1,1)-Markov model can combine the advantages of small samples predicted by $\mathrm{GM}(1,1)$ model and the advantages of Markov model response fluctuation to improve the prediction accuracy. Varicella data was small and volatility, and good prediction results were obtained by using GM(1,1)-Markov model. GM(1,1)-Markov model can be used, improved and popularized in the prediction of similar types of data in the future.

\section{Abbreviations}

$\mathrm{P}=$ the probability of small error, $\mathrm{C}=$ the posterior difference ratio $\quad \mathrm{CDC}=$ Centers for Disease Control and Prevention, GM $(1,1)=$ grey model first-order one variable, VZV= varicella-zoster virus

\section{Declarations}

\section{Ethics approval and consent to participate}

Not application

\section{Consent for publication}

Not application 


\section{Acknowledgements}

Not application

\section{Availability of data and materials}

The data that support the findings of this study are available from Dalian Centers for Disease Control and Prevention (CDC) but restrictions apply to the availability of these data, which were used under license for the current study, and so are not publicly available. Data are however available from the authors upon reasonable request and with permission of Dalian Centers for Disease Control and Prevention (CDC).

\section{Competing interests}

The authors declare that they have no competing interests.

\section{Funding}

Not application

\section{Authors' contributions}

Author1.CT:Conducted the research, did data entry $\llbracket$ processed and analysis the data \and was a major contributor in writing the manuscript.

Author2.BY: Provided the original data.

All authors read and approved the final manescript.

\section{Acknowledgements}

Not application

\section{References}

1. Gershon, A. A. et al. Varicella zoster virus infection. Nature reviews. Disease primers 1, 15016, doi:10.1038/nrdp.2015.16 (2015).

2. Ahn, K. H. et al. Congenital varicella syndrome: A systematic review. Journal of obstetrics and gynaecology: the journal of the Institute of Obstetrics and Gynaecology 36, 563-566, 
doi:10.3109/01443615.2015.1127905 (2016).

3. Amlie-Lefond, C. \& Gilden, D. Varicella Zoster Virus: A Common Cause of Stroke in Children and Adults. Journal of stroke and cerebrovascular diseases: the official journal of National Stroke Association 25, 1561-1569, doi:10.1016/j.jstrokecerebrovasdis.2016.03.052 (2016).

4. Bernal, J. L., Hobbelen, P. \& Amirthalingam, G. Burden of varicella complications in secondary care, England, 2004 to 2017. Euro surveillance: bulletin Europeen sur les maladies transmissibles = European communicable disease bulletin 24, doi:10.2807/1560-7917.es.2019.24.42.1900233 (2019).

5. Xu, Q. \& Xu, A. Epidemiological analysis of varicella in China and advance in immunity prevention for varicella vaccine. Chinese Journal of New Drugsa 21, 1093-1098 (2012).

6. US children to get a second dose of chickenpox vaccine. Chinese JOurnal of General Practitioners 6 , 603 (2007).

7. Leung, J., Broder, K. R. \& Marin, M. Severe varicella in persons vaccinated with varicella vaccine (breakthrough varicella): a systematic literature review. Expert review of vaccines 16, 391-400, doi:10.1080/14760584.2017.1294069 (2017).

8. Jiang, C. Epidemiological characteristics of varicella patients in Xuzhou, 2010-2018. Modern Preventive Medicine 47, 2328-2331+2341 (2020).

9. Pan, X., Ma, R., Fang, T., Xu, G. \& Xu, H. Epidemiology of varicella in Ningbo city of Zhejiang province, 2012-2016. Chinese Journal of Vaccines and Immunization 23, 302-305 (2017).

10. Zeng, J., Yu, X., Liu, J., Tan, A. \& Xie, D. Analysis on epidemiological characteristics of varicella in Neijiang City of Sichuan Province from 2009 to 2018. Journal of Medical Pest Contro/ 36, 400-403 (2020).

11. Zhang, H. Diagnosis and treatment of chickenpox. World Health Digest 09, 229-230, doi:10.3969/j.issn.1672-5085.2012.18.229 (2012).

12. Yang, Y., Geng, X., Liu, X., Wang, W. \& Zhang, J. Association between the incidence of varicella and meteorological conditions in Jinan, Eastern China, 2012-2014. BMC infectious diseases 16, 179, doi:10.1186/s12879-016-1507-1 (2016).

13. Deng, J. Basic Methods of Grey System Chinese-English Second Edition. (Huazhong Institute of Technology Press, 2005).

14. Fu, B. \& Wang, A. Application of Markov Chain in Market Forecast. CO-Operative Economy \& Science, 56-57, doi:10.3969/j.issn.1672-190X.2017.01.027 (2017).

15. Dang, S. L. Y. Grey System Theory and Application Fifth Edition. (Beijing: Science Press, 2010).

16. Tang, T. \& Li, L. Prediction of Container Throughput of Shanghai Port Based on Grey Markov Model. Logistics Sci-tech 43, 105-108,114 (2020).

17. Du, B. Research on Pilot Demand Quantity Prediction and Team Construction Based on Grey Markov Method, DalianMaritimeUniversity, (2017). 
18. Song, X. Study on Coal Production Prediction Based on Gray Markov Model. Mineral Engineering Research 34 (2019).

19. Bai, S. Epidemiological characteristics and prediction of varicella in Shenyang from 2006 to 2018. Journal of Tropical Medicine 19, 1564-1566 (2019).

20. Li, X. Analysis on the Epidemiologic Characteristics of varicella in Qingdao from 2007 to 2016, Qingdao University, (2018).

21. LAFORET, E. G. \& LYNCH, C. L. Multiple congenital defects following maternal varicella; report of a case. The New England journal of medicine, 534-537 (1947).

22. Song, Y. Consequences of varicella in pregnancy or perinatal period. Chinese Journal of Experimental and Clinical Virology 30 (2016).

23. Lv, T. \& Yang, H. Varicella-zoster virus infection during pregnancy. Chinese Journal of Medicine, 1821 (2008).

24. Peng, Y., Kong, D., Wang, P., Lu, S. \& Yang, X. Epidemiological characteristics of chicken-pox in Wuhan city, 2009-2018. Practical Preventive Medicine 27, 330-332 (2020).

25. Zhang, T. Pre-hospital emergency treatment demand for cardiovascular and cerebrovascular disease in Urumqi based on grey model and time series model: Prediction and analysis. Chinese Journal of Disaster Medicine 7 (2019).

26. Wang, Y.-w., Shen, Z.-z. \& Jiang, Y. Comparison of ARIMA and GM(1,1) models for prediction of hepatitis B in China. Public Library of Science 13 (2018).

27. Cheng, G. Impacts of Different Dimensions in Gray Model on Model Precision. Standardization for Surveying and Mapping 34 (2018).

28. Kai, Z. \& Ya-dong, L. I. The mining subsidence based on the innovation $\mathrm{GM}(1,1)$ model. Beijing Surveying and Mapping, 31-33, doi:10.3969/j.issn.1007-3000.2013.02.008 (2013).

29. Yi, J. et al. Application of Three Kinds of Model in Forecasting Prevalence of Pulmonary Tuberculosis. Chinese General Practice 15, 1495-1497, doi:10.3969/j.issn.1007-9572.2012.13.022 (2012).

30. Shi, D. Study on prediction of occupational diseases based on grey GM(1,1)-Markov model. Journal of Safety Science and Technology 13 (2017).

31. Pei, Y. Predictive analysis of bridge condition by improved Grey Markov model, Heibei engineering university (2018).

\section{Figures}




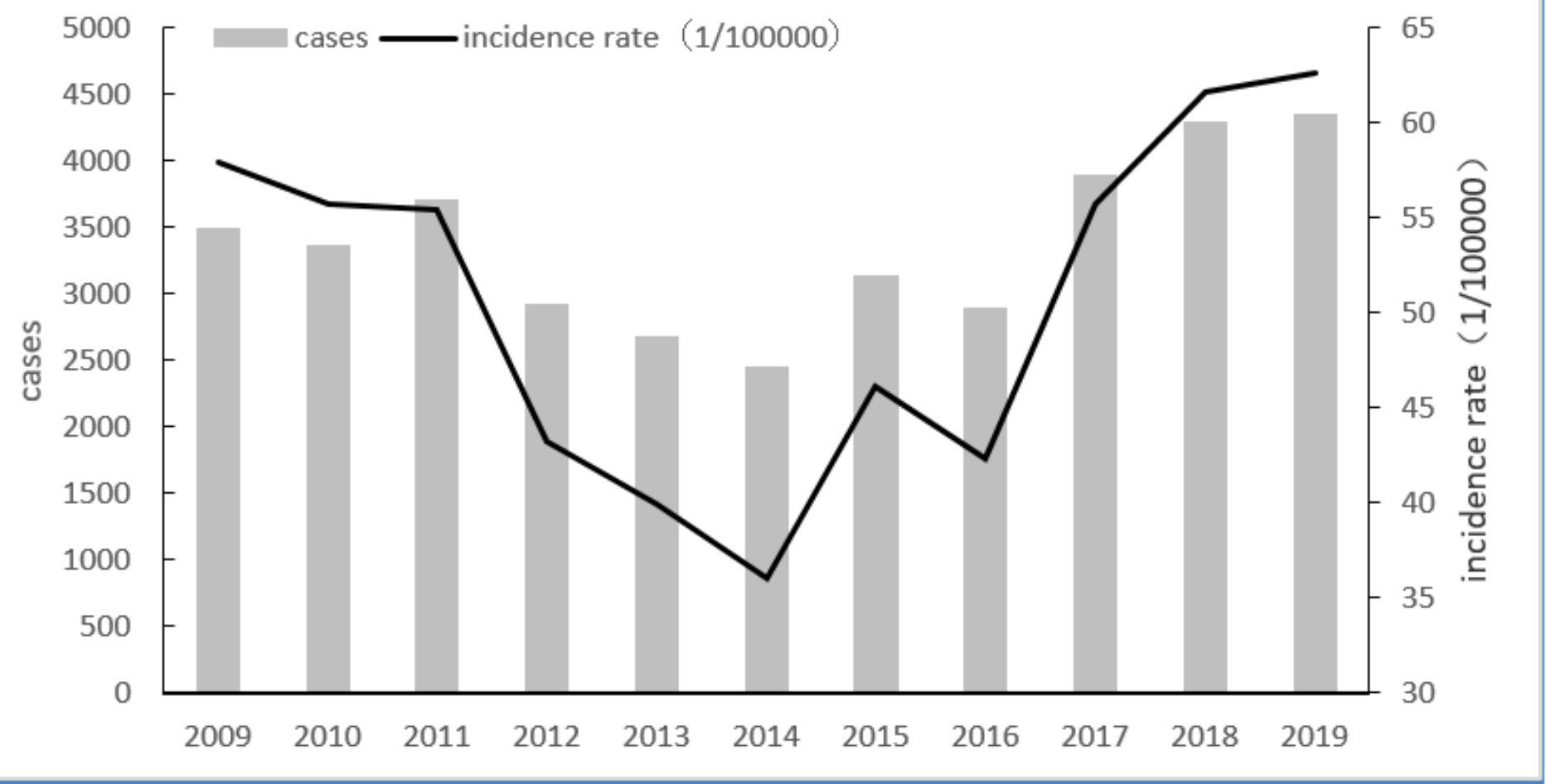

Figure 1

The cases and incidence rate of varicella in Dalian from 2009 to 2019

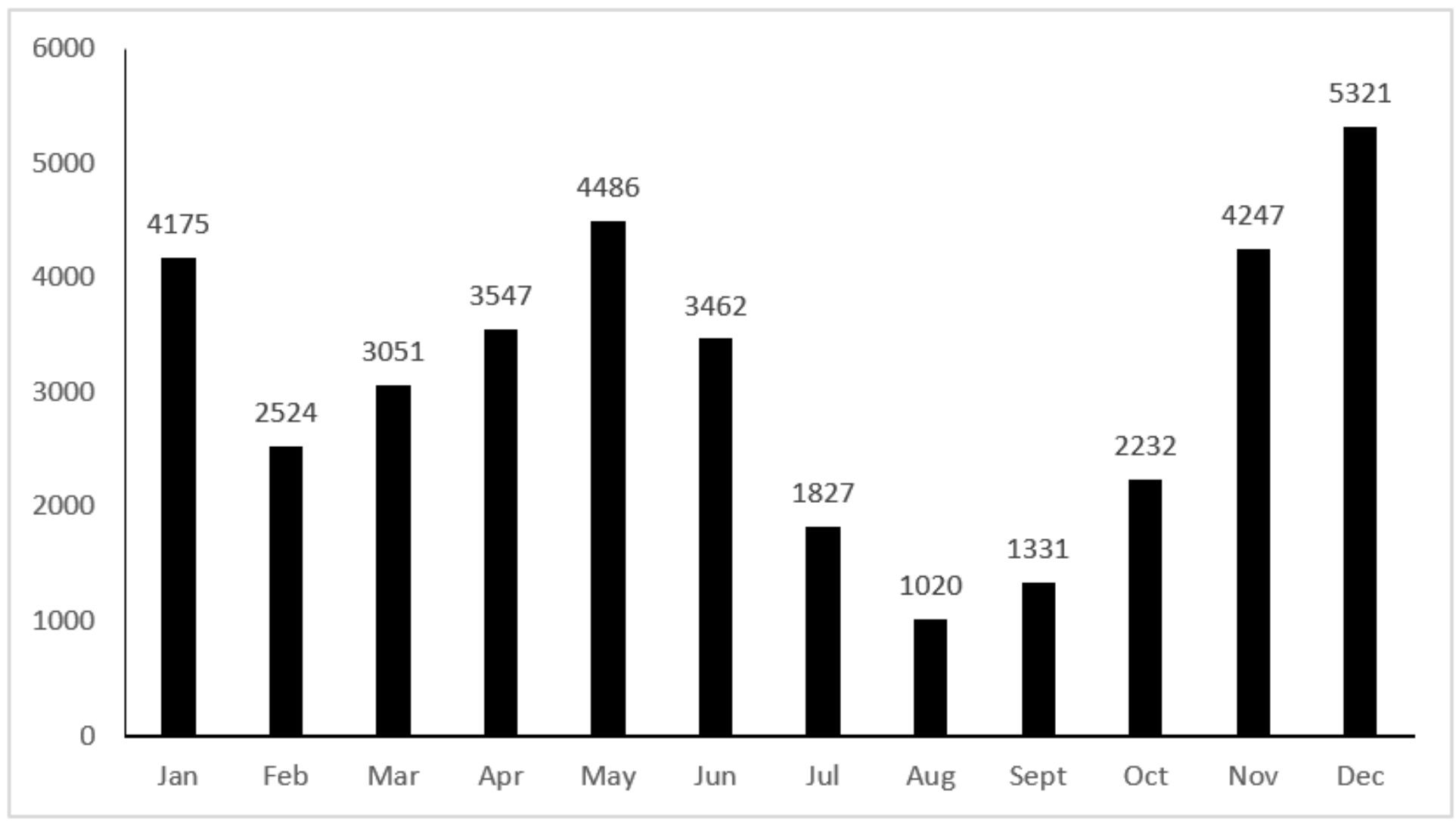


Figure 2

Month distribution of varicella cases in Dalian from 2009 to 2019

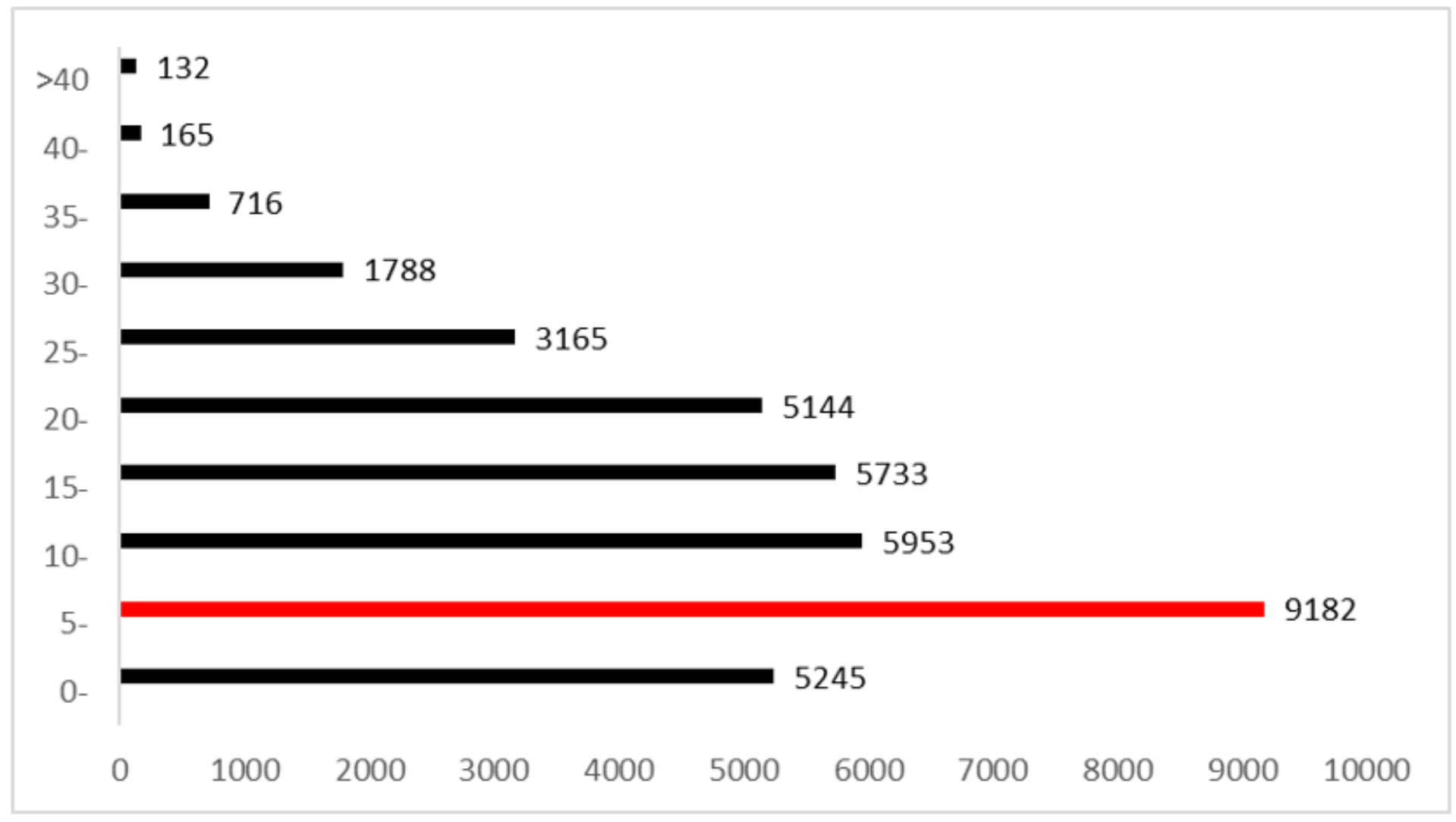

\section{Figure 3}

The varicella cases age distribution in Dalian from 2009 to 2019 


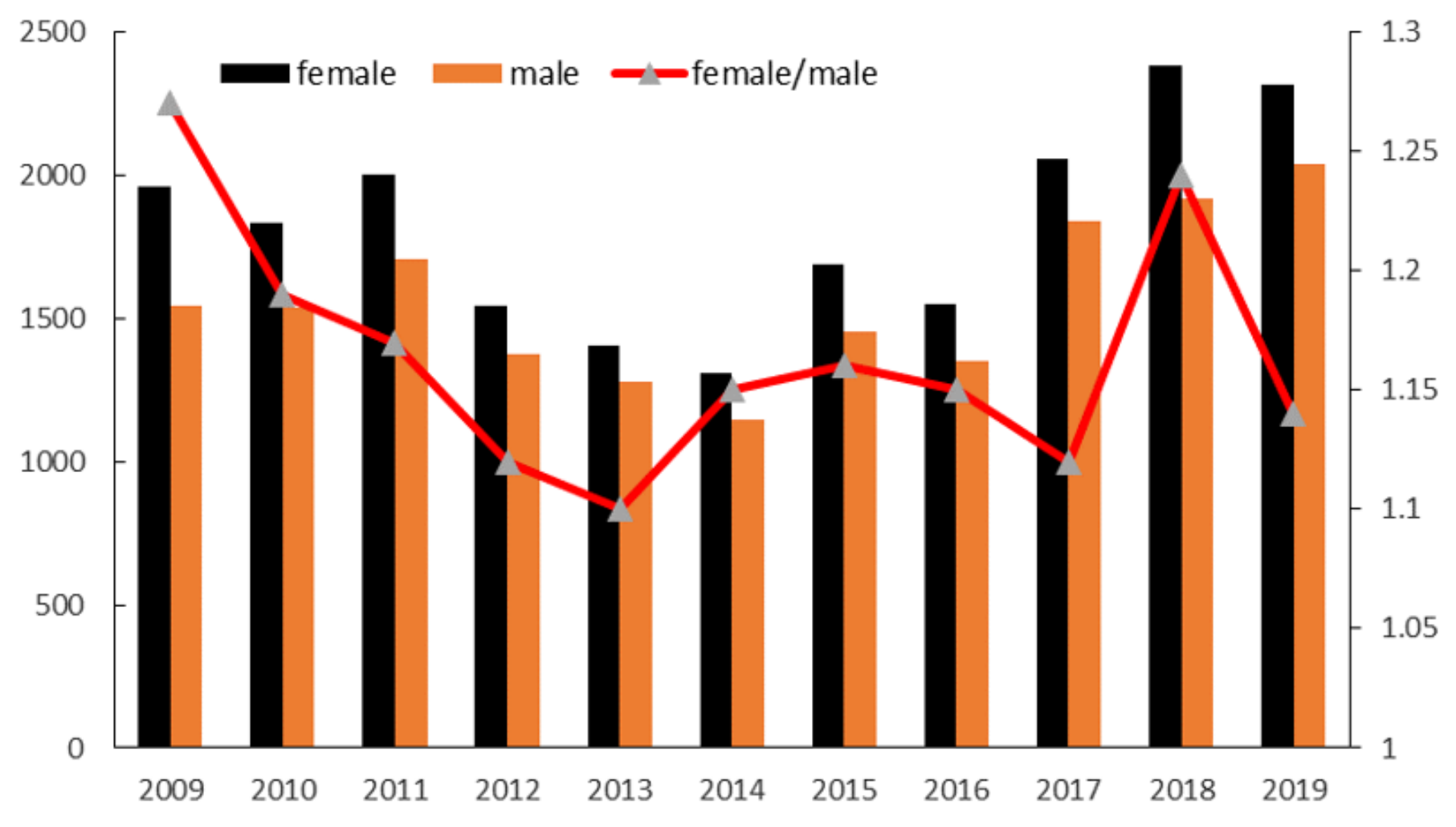

Figure 4

Total cases and gender distribution of varicella in Dalian from 2009 to 2019 


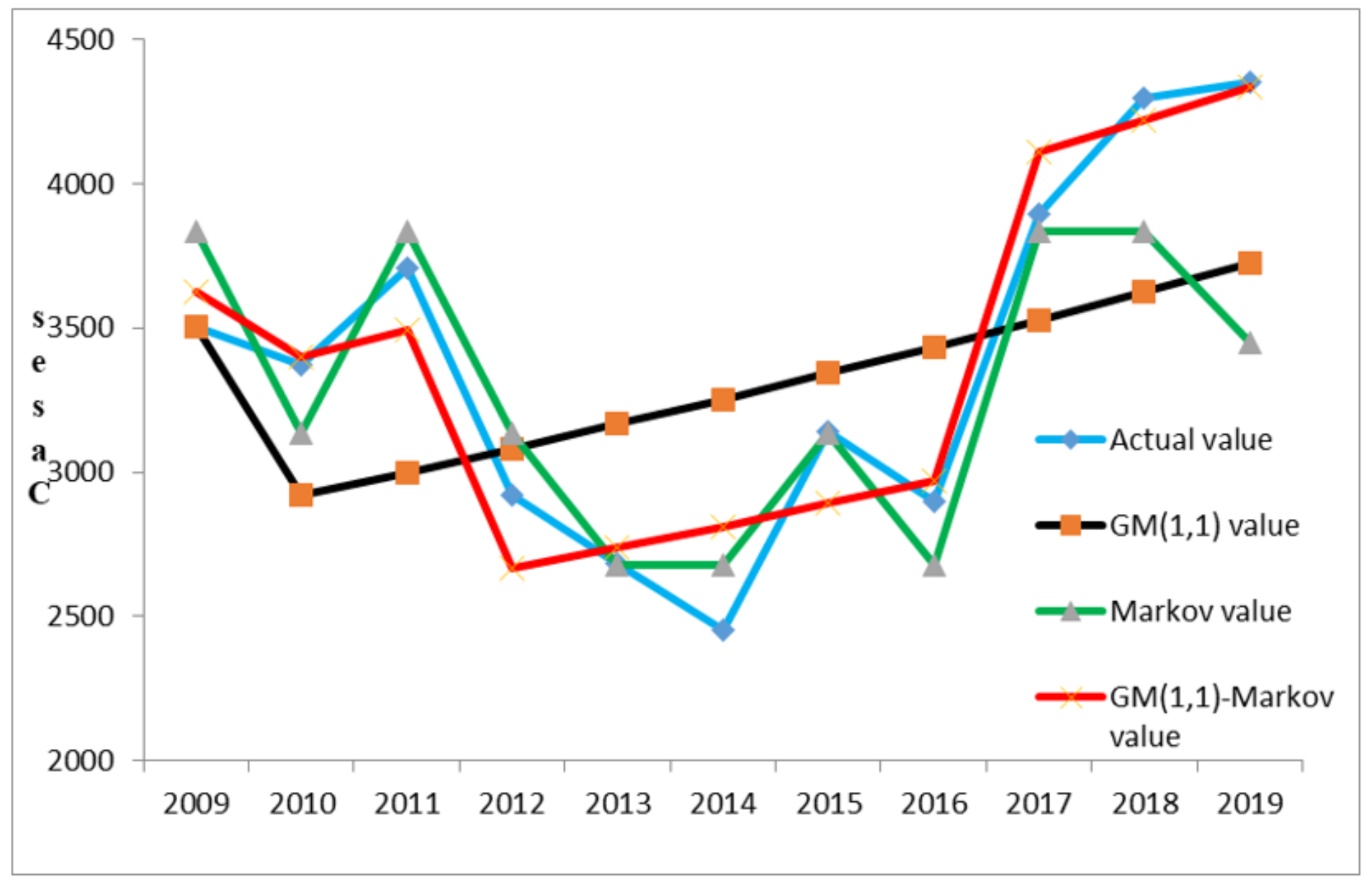

Figure 5

Comparison between prediction value and actual value

\section{Supplementary Files}

This is a list of supplementary files associated with this preprint. Click to download.

- SupplementaryMaterial.docx 